\title{
Cost awareness among healthcare professionals at a South African hospital: A cross-sectional survey
}

\author{
G D Nethathe, ${ }^{1,2} \mathrm{MB}$ ChB, DA (SA), FCA (SA), MMed (Anaes), FANZCA, Cert Crit Care (SA), PGDip Health Science Education; \\ S Tshukutsoane, ${ }^{2}$ BCur Hons; K J Denny, ${ }^{3} \mathrm{PhD}, \mathrm{MBBS}, \mathrm{BSc}$ \\ ${ }^{1}$ Department of Anaesthesia and Perioperative Medicine, Royal Brisbane and Women's Hospital, Brisbane; and Faculty of Medicine, \\ University of Queensland, Brisbane, Australia \\ ${ }^{2}$ Division of Critical Care, School of Clinical Medicine, Faculty of Health Sciences, University of the Witwatersrand, Johannesburg, South Africa \\ ${ }^{3}$ Department of Intensive Care, Royal Brisbane and Women's Hospital, Brisbane, Australia
}

Corresponding author: G D Nethathe (gladness.nethathe@wits.ac.za)

Background. Financial cost is a recognised cause of lack of access to adequate healthcare in South Africa (SA). Data describing the SA healthcare professional (HCP)'s awareness of costs are scant. Their increased awareness of healthcare costs may improve efficacy and reduce wasteful expenditure.

Objective. To assess SA HCP's knowledge of healthcare costs, identify factors that influence cost awareness, and to determine if surveyed HCPs received training related to cost management during their studies or at any stage during their practice.

Methods. This cross-sectional survey was conducted by means of a standardised questionnaire. HCPs working at a major tertiary academic hospital were asked to answer an anonymous standardised questionnaire aimed at determining their awareness of the costs of commonly requested hospital items and tests. Cost accuracy was determined by assessing the log deviation of the estimated cost from true cost, with values $>0$ and $<0$ representing overestimates and underestimates, respectively. Cost estimations were considered correct if the absolute value of the log deviation was $<0.2$. Participants' attitudes towards the potential impact of the availability of cost information on their practice were assessed. Results. The overall cost estimation of accuracy was low (mean 0.60; standard deviation 1.99) and differed widely between items. Cheaper items were more likely to be overestimated and expensive items to be underestimated. The majority of participants indicated that cost awareness education was not part of their training or practice (84.5\%) and that they would like cost information to be made readily available (92.2\%). Eighty-four percent of participants were of the opinion that cost information would not negatively affect patient care.

Conclusion. The use of percentage deviation from true cost as a method of assessing cost awareness creates a bias towards overestimation, which is more relevant for cheap items, as larger overestimates are more common for these items. We propose the use of log deviation of the estimated cost from the true cost as a method of assessing cost estimation accuracy. HCPs have a limited understanding of the costs of disposables, tests and drugs commonly used in their practice and would prefer that cost information be made readily available to them. Attention should be paid to improving cost awareness among HCPs working at SA hospitals.

S Afr Med J 2017;107(11):1010-1014. DOI:10.7196/SAMJ.2017.v107i11.12513

Financial cost is a recognised cause of lack of access to adequate healthcare in South Africa (SA). ${ }^{[1,2]}$ Higher costs have implications with regard to the standard of care and have been shown to negatively affect patient outcomes. ${ }^{[3]}$ Higher costs also have wider implications, including influencing healthcare decisions taken by households. ${ }^{[4,5]}$ It is notable that SA's level of healthcare spending is higher than that in other countries of a similar developmental status and some high-income countries, while health status indicators are much worse, suggesting that equitable and efficient resource utilisation is a challenge.$^{[6]}$ Healthcare professionals (HCPs) are ideally positioned to act as gatekeepers to ensure the effective use of resources. Numerous studies have demonstrated improved cost containment by HCPs when cost information is made available. ${ }^{[7,8]}$ Tierney et al. ${ }^{[7]}$ studied the effect of displaying the cost of diagnostic tests ordered at microcomputer workstations on the number of tests ordered by physicians in an academic primary care medical practice. The displaying of costs led to a reduction in the number and cost of tests ordered; this effect did not persist after the intervention was discontinued. Additionally, HCPs often have a limited understanding of the true cost of items used in their practice, consistently underestimating expensive items and overestimating less expensive ones. ${ }^{[9,10]} \mathrm{A}$ survey of internal medicine residents at an academic tertiary centre showed that participants poorly estimated the cost of diagnostic tests. ${ }^{[8]}$ The percentage of correct estimates, as defined by those within $25 \%$ of the actual cost, ranged from $3 \%$ for a D-dimer test to $39 \%$ for magnetic resonance imaging of the brain. Participants also indicated that they would like to improve their cost information knowledge. ${ }^{[8]}$

There is a gap in the SA literature with regard to HCPs' cost awareness, which we aim to address. The objective of our study was to assess SA HCPs' knowledge of healthcare costs and to identify factors that influence cost awareness. Specifically, we sought to determine whether HCPs at a major tertiary academic hospital were aware of the cost of 15 selected drugs, medical products, disposables and investigations commonly used in their practice, and whether there are differences in cost estimation accuracy between groups of HCPs according to levels of training, specialty and involvement in private practice. We also sought to determine whether surveyed HCPs received training relating to cost management during their studies or at any stage during their practice.

\section{Methods}

This was a single-centre cross-sectional survey conducted by means of a standardised questionnaire. The study was conducted at four clinical departments at Chris Hani Baragwanath Academic Hospital 
(CHBAH), Johannesburg, SA. This tertiary academic hospital is affiliated to the University of the Witwatersrand, Johannesburg.

The questionnaire assessed knowledge of the cost of 15 items commonly used in clinical practice. The items included 4 drugs, 5 intravenous fluids, 3 disposable items and 3 laboratory tests. HCPs were asked about prior education or training relating to cost awareness, as well as their attitudes towards the availability of cost information and its influence on patient care.

Cost was defined as the 'single exit price', which was the current price set for $\mathrm{CHBAH}$ by the manufacturer or importer of a medicine or disposable combined with the logistics fee and value-added tax. The National Health Laboratory Service provided the cost of the laboratory investigations. Cost estimations were considered correct if the absolute value of the log deviation was $<0.2$.

\section{Ethical approval}

Ethical approval was obtained from the Human Research Ethics Committee (medical) of the University of the Witwatersrand before commencement of the study (ref. no. M150337). Participants were de-identified to ensure anonymity. Participating HCPs included house officers, registrars, specialists, clinical nurses and nursing assistants employed in the departments of anaesthesia, intensive care, obstetrics and gynaecology, and general surgery.

\section{Statistical analysis}

Data analysis was conducted using the open-source software $\mathrm{R}$ (3.2.2). The mean log deviation and standard deviation (SD) of the estimated cost of each item were calculated for age, gender, level of training, number of years in clinical practice, various groups of HCPs (intensive care, general surgery, obstetrics and gynaecology, anaesthesia) and private practice involvement. Log deviation was taken as the natural logarithm of the estimate divided by the true cost.

Accuracy was assessed by calculating the mean, median, SD, confidence interval (CI) and interquantile ranges of the log deviation per group. Log deviations with an absolute value of $<0.2$ were regarded as accurate. The frequency of correct estimates, underestimates and overestimates was calculated. Accuracy was compared using box plots between level of training, number of years in healthcare service, various groups of HCPs according to department (intensive care, general surgery, obstetrics and gynaecology, anaesthesia) and private practice involvement. Differences in means were investigated using analysis of variance (ANOVA). Levels of $p<0.05$ were accepted as significant.

\section{Results}

Of the 587 questionnaires distributed, 271 were received (response rate $46.16 \%$ ). Of these, 26 were excluded ( 13 were completed by medical students, 4 by members of departments not part of the study sample, and 9 did not answer 7 or more cost estimation questions). The demographics of the respondents are shown in Table 1.

The mean period of time in clinical practice was 14.25 (range 0.00 $42.00)$ years. Ninety-seven (39.6\%) participants stated involvement in private practice, $143(58.4 \%)$ were not involved and $5(2.0 \%)$ did not respond to this question. The average time of involvement in private practice was 6.57 (median 4.00; range $0.01-30.00$ ) years. A total of $152(62.04 \%)$ did not respond to this question.

Overall, cost estimation accuracy was low (median 0.60; SD 1.99) and differed widely between items. Cheaper items were more likely to be overestimated and expensive items to be underestimated (Fig. 1).

There were no significant differences in cost accuracy between departments $(p=0.078)$, position in department $(p=0.167)$ or number of years in clinical practice $(p=0.187)$. Similarly, involvement in private practice was not significantly associated with a difference
Table 1. Demographics of surveyed healthcare practitioners $(N=245)$

\begin{tabular}{ll}
\hline Demographics & $\boldsymbol{n}(\%)$ \\
\hline Gender & $38(15.5)$ \\
Female & $128(52.2)$ \\
Male & $79(32.2)$ \\
Not stated & \\
Department & $23(9.4)$ \\
Anaesthesia & $78(31.8)$ \\
General surgery & $118(48.1)$ \\
Intensive care & $24(9.8)$ \\
Obstetrics and gynaecology & $2(0.8)$ \\
Not stated & \\
Position in department & $23(9.3)$ \\
Intern & $15(6.1)$ \\
Medical officer & $8(3.2)$ \\
Nurse assistant & $114(46.5)$ \\
Professional nurse & $26(10.6)$ \\
Registrar & $31(12.6)$ \\
Specialist/consultant & $24(9.8)$ \\
Staff nurse & $3(1.2)$ \\
Other & $1(0.4)$ \\
Not stated & \\
Involvement in private practice & $97(39.6)$ \\
Yes & $143(58.4)$ \\
No & $5(2.0)$ \\
Not stated & \\
& \\
\hline &
\end{tabular}

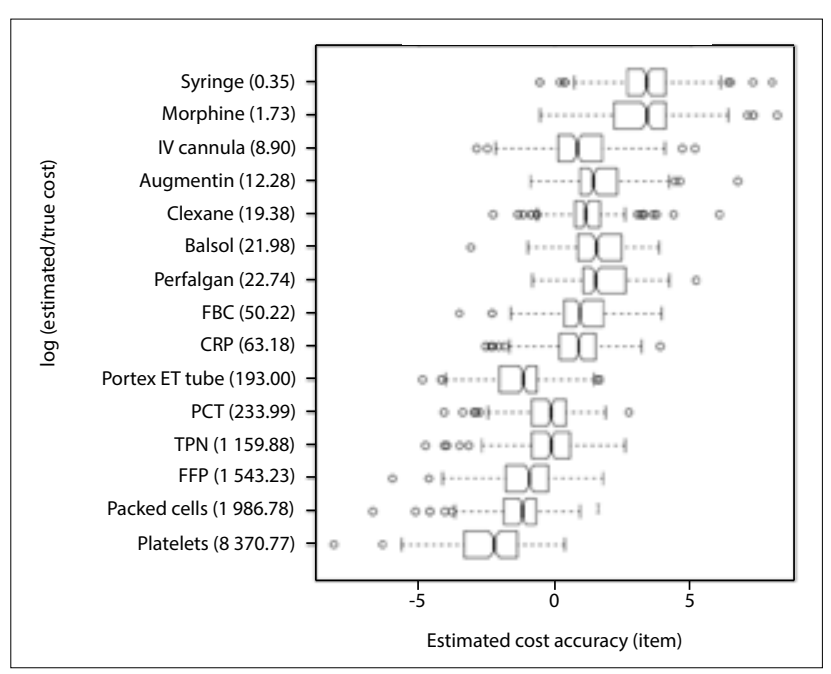

Fig. 1. Estimated cost accuracy by item, showing log of estimated cost over true cost and estimated cost accuracy by item. (IV = intravenous; $F B C=$ full blood count; $C R P=C$-reactive protein; $E T=$ endotracheal tube; $P C T=$ procalcitonin; $T P N=$ total parenteral nutrition; $F F P=$ fresh-frozen plasma . Median values (dashed line); $50 \%$ percentile values (box outline); 90 th percentile values (whiskers); and outlier values (open circles). Values $>0$ represent overestimations, and those $<0$ underestimations.)

in accuracy of cost estimations $(p=0.934)$. Factors influencing cost accuracy are illustrated in Table 2. 
Table 2. Cost accuracy estimations per position in department, department, number of years in clinical practice, and involvement in private practice $(N=245)^{*}$

\begin{tabular}{|c|c|c|c|c|c|}
\hline & $\begin{array}{l}\text { Accurate } \\
(-0.2-0.2)\end{array}$ & $\begin{array}{l}\text { Underestimation } \\
(\leq-0.2)\end{array}$ & $\begin{array}{l}\text { Overestimation } \\
(\geq 0.2)\end{array}$ & Mean (SD) & $p$-value \\
\hline \multicolumn{6}{|l|}{ Position in department, $\%$} \\
\hline Intern & 11.01 & 29.27 & 59.71 & $0.73(1.7)$ & 0.167 \\
\hline Medical officer & 10.00 & 26.81 & 63.18 & $0.61(1.7)$ & \\
\hline Registrar & 8.50 & 32.98 & 58.50 & $0.58(1.6)$ & \\
\hline Specialist & 9.54 & 30.80 & 59.65 & $0.66(1.6)$ & \\
\hline Nurse assistant & 4.20 & 37.81 & 57.98 & $0.41(2.4)$ & \\
\hline Professional nurse & 6.84 & 34.50 & 58.65 & $0.55(2.1)$ & \\
\hline Staff nurse & 4.87 & 34.67 & 60.45 & $0.78(2.4)$ & \\
\hline Other & 8.88 & 37.77 & 53.33 & $0.03(2)$ & \\
\hline \multicolumn{6}{|l|}{ Department, \% } \\
\hline Anaesthesia & 10.05 & 31.65 & 58.28 & $0.44(1.5)$ & 0.078 \\
\hline General surgery & 7.85 & 33.94 & 58.20 & $0.54(2.1)$ & \\
\hline Intensive care & 7.13 & 33.58 & 59.28 & $0.63(2.1)$ & \\
\hline Obstetrics and gynaecology & 7.79 & 29.24 & 62.95 & $0.79(1.8)$ & \\
\hline \multicolumn{6}{|l|}{ Clinical practice (years), $n(\%)$} \\
\hline $0-10$ & $179(8.04)$ & $698(31.37)$ & $1348(60.58)$ & $0.64(1.9)$ & 0.187 \\
\hline $11-20$ & $31(7.52)$ & $153(37.13)$ & $228(55.33)$ & $0.67(1.9)$ & \\
\hline$\geq 21$ & $38(7.40)$ & $171(33.52)$ & $303(50.06)$ & $0.51(2.1)$ & \\
\hline \multicolumn{6}{|c|}{ Private practice involvement, $n$ (\%) } \\
\hline Yes & $102(7.15)$ & $476(33.38)$ & $848(59.47)$ & $0.60(2)$ & 0.934 \\
\hline No & $173(8.14)$ & $698(32.86)$ & $1253(58.99)$ & $0.59(2)$ & \\
\hline
\end{tabular}

Respondents were also asked if they used any other medical product or investigation that was not part of the listed items of which they knew the price. Thirty-eight (15.51\%) indicated 'yes', 210 (85.71\%) indicated 'no' and 6 (2.44\%) did not respond to this question. Thirty-nine (15.91\%) of those surveyed indicated that they had received healthcare cost management education at any point during their training, while the majority $(n=209 ; 85.30 \%)$ indicated that they had not. Six participants $(2.44 \%)$ did not respond to this question.

HCPs were asked whether they require the cost information of drugs, equipment and disposables to be readily available. The majority requested more readily available information regarding the cost of such health resources (yes: $n=226,92.24 \%$; no: $n=23,9.38 \%$; not stated: $n=5 ; 2.04 \%)$. Furthermore, the majority of respondents felt that knowledge of cost information of medical consumables and services would improve both resource management (98.36\%) and patient care (83.67\%). Of those surveyed, $16.73 \%$ thought that knowledge of cost information of medical products and services would negatively impact patient care.

\section{Discussion}

The results of this study are in keeping with those of other studies on cost awareness of HCPs that have found cost estimation accuracy to be low. ${ }^{[3,9-12]}$ In a systematic review by Allan et al., ${ }^{[3]}$ investigating doctors' knowledge of the cost of medications, a low cost estimation accuracy was found, with $31 \%$ of estimates being within $20 \%$ or $25 \%$ of the true cost.
A survey conducted by Bade et al. ${ }^{[9]}$ in three New Zealand hospitals, showed that only $18.6 \%$ of estimates fell within $\pm 25 \%$ of true cost. Cost (51.2\%) was underestimated in their study. A survey conducted among anaesthetic staff in a UK practice showed more accurate responses than other studies. ${ }^{[13]}$ The definition of accuracy was not stated in their methodology. Forty-seven percent of estimates were within $50 \%$ of the true cost and $75 \%$ within $100 \% .{ }^{[13]}$ The cost of expensive items was underestimated and that of cheap items was consistently overestimated. ${ }^{[13]}$ HCPs also indicated that they had limited training in cost awareness measures, would like cost information to be readily available, and that such information would possibly improve patient outcomes.

The trend towards underestimating the prices of expensive drugs and overestimating those of less expensive drugs, as evident in our study, has been found in other similar studies. ${ }^{[3,11]}$ This finding is not surprising, considering the method that is often used to estimate cost accuracy, i.e. estimated price or real price deviation from true cost. ${ }^{[9,10,12]}$ The overestimation of inexpensive drugs and equipment is inherent in studies that use percent deviation from true cost in assessing cost accuracy. ${ }^{[9,10,12]}$ For example, a drug that costs ZAR1, but is overestimated by a participant to cost ZAR2, is assessed as being $100 \%$ overestimated by such a method (albeit still correctly assessed as being cheap). However, an expensive item that costs ZAR100 000 might be estimated to cost ZAR150 000 by a study participant, with a resultant $50 \%$ overestimation. Firstly, it is unusual for a cost estimation of an item to be a negative number. Secondly, participants may overestimate an item by a magnitude of $300 \%$, but 
are unlikely to underestimate such an item as costing $300 \%$ less, i.e. a negative number. One can underestimate by a maximum of $100 \%$, but overestimate by a larger factor, which emphasises overestimation, especially for cheap items. The use of percentage deviation overquantifies overestimates and under-quantifies underestimates (which can be at most $100 \%$; overestimates can be $\geq 100 \%$ ). Hence, it is more appropriate to use log deviation, as has been done in this study. Log deviation quantifies the scale of mis-estimations and minimises this effect by measuring the scale factor of the estimations.

With regard to factors affecting cost awarenss, a study by Glickman et al. ${ }^{[11]}$ showed that younger rather than older physicians were more likely to make correct estimates, and specialists in internal medicine were less likely to make correct estimates than those from other disciplines. Allan et al.' $\mathrm{s}^{[3]}$ systematic review of physician awareness of drug cost, by investigating doctors' knowledge of the cost of medications and factors influencing cost awareness, not only showed a low cost estimation accuracy but also that high-cost items were more accurately estimated than inexpensive ones. Doctors thought that cost information would improve their prescribing, but these data were not accessible. ${ }^{[3]}$ Three studies in this review found cost estimations by non-academic physicians more accurate than those by academic physicians. ${ }^{[3]}$

In the survey conducted by Bade and Hoogerbrug, ${ }^{[9]}$ no significant differences in accuracy were found between consultants, registrars and house officers or between consultants working in private and those in both private and public practice.

The current study found no significant differences in cost estimation accuracy between departments based on number of years in clinical practice.

Numerous studies have found that there is no difference in cost estimation accuracy between HCPs at various levels of training and experience, finding no such differences among medical students, residents, faculty physicians, specialists and physicians. ${ }^{[11,14,15]} \mathrm{We}$ found that private practice involvement does not significantly influence cost estimation accuracy.

Our study highlights that HCPs perceive that they do not have sufficient training relating to cost awareness. Participants indicated that they would like cost information to be readily available and that knowledge thereof would not negatively affect patient care.

Educational interventions aimed at the teaching of cost consciousness have yielded conflicting results. ${ }^{[15,16]}$ Radiological cost related to abdominal imaging was significantly reduced in one study after an educational cost awareness intervention, whereas readmission risk coupled with a non-significant cost difference between the intervention and control group was evident in another study that aimed to reduce costs by a similar educational intervention. ${ }^{[15,16]}$ This highlights that educational interventions aimed at reducing costs have to be carefully structured before implementation and that they may not necessarily lead to the overall reduction of costs. A simple intervention might be to provide and make readily accessible cost information relating to tests, disposables and equipment; the effect of this has been shown to persist during the period that the cost information is available. ${ }^{[7]}$ Awareness of the lack of knowledge of healthcare-associated costs and the desire to improve such knowledge highlight the progressive attitudes towards cost awareness and cost consciousness in this group. However, despite working in a resource-limited setting, HCPs in our study demonstrated poor estimation of cost accuracy patterns seen in studies done in more resource-abundant regions in Europe, Australasia and North America. ${ }^{[3,9,13,17-19]}$

Cost accuracy has remained poor in almost all studies assessing cost awareness, which may be due to poor education regarding the cost of healthcare consumables. ${ }^{[3]}$ We hypothesise that a mathematical error consistent in most studies assessing cost awareness - the use of the percent deviation from the mean - contributes to this. Therefore, we employed the log deviation. Our results, however, demonstrate poor cost awareness, as has been observed consistently with previous studies. $^{[3,9,13,17-19]}$

\section{Study limitations}

As this was a single-centre study, the study population may not be representative of HCPs in SA. The cost of expensive medical equipment or radiological investigations was not assessed because of logistical reasons. Various departments may have access to and thus familiarity with different equipment owing to the nature of their work; this study included a wide spectrum of HCPs. The cost of radiological investigations is not standard and also tends to differ, depending on the urgency of the request and the human resource and labour costs involved. We chose commonly used items and those that were unlikely to vary between departments in order to include various HCPs, avoiding bias with regard to occupation-related familiarity with specific equipment.

The interpretation of cost estimation accuracy differs among studies. Percentage deviation from true cost is often used to assess accuracy. If the cost estimation of an item or test is considered 'accurate' when the estimate is $\pm 25 \%$ of the true cost, the results will be errors of a small magnitude in true cost, but larger errors in terms of percentage. The latter are then highlighted as grossly deviant from the true cost. For example, an item of ZAR1 being overestimated as ZAR2, represents a ZAR1 difference from true cost, but a $100 \%$ deviation from true cost. However, the participant is still aware that the item falls into the 'cheap' category. This may not be economically relevant, as the item would still fall into the cheap category. We hypothesise that this is the reason that cheap items are often overestimated in cost awareness studies. Therefore, we used the more logical approach of $\log$ deviation. Although more mathematically correct, this is not in alignment with most (if not all) medical studies of cost awareness.

\section{Conclusion}

HCPs' estimations of cost were inaccurate. The majority indicated that they had not received adequate training with regard to cost and that they would like cost information to be readily available. This is significant, as HCPs may help to reduce healthcare expenditure. Increasing cost awareness may result in effective resource management. It is hoped that the findings of this study will help to inform cost awareness programmes and encourage the use of log deviation of true cost over estimated cost in the assessment of cost accuracy estimation.

Acknowledgements. Dr U Kirchner recommended using absolute log deviation in the assessment of cost accuracy estimations and assisted with the analysis and interpretation of the data. We would like to thank the departments of anaesthesia, general surgery, intensive care and obstetrics and gynaecology at $\mathrm{CHBAH}$ for participating in the study. We thank Prof. L R Mathivha for her assistance with reviewing the proposal, Dr S Vally for her assistance with the true cost of laboratory tests, and Drs E Buga and P Mogane for assisting with data collection.

Author contributions. GDN: conceived and designed the study, analysed and interpreted the data, and prepared the article for publication; ST: assisted with proposal development and ethics submission, investigated the true cost of the medications, distributed the survey, and collected and 
captured the data; and KJD: supervised the completion of the project and assisted with the preparation and revision of the article for publication.

Funding. None.

Conflicts of interest. None.

1. Harris B, Goudge J, Ataguba JE, et al. Inequities in access to healthcare in South Africa. J Public Health Policy 2011;32(Suppl 1):S102-S123. https://doi.org/10.1057/jphp.2011.35

2. Macha J, Harris B, Garshong B, et al. Factors influencing the burden of healthcare financing and the distribution of healthcare benefits in Ghana, Tanzania and South Africa. Health Policy Plan 2012;27(Suppl 1):i46-i54. https://doi.org/10.1093/heapol/czs024

. Allan GM, Lexchin J, Wiebe N. Physician awareness of drug cost: A systematic review. PLoS Med 2007;4(9):e283. https://doi.org/10.1371/journal.pmed.004028

4. Van Rensburg HC. South Africas's protracted struggle for equal distribution and equitable access - still not there. Hum Resource Health 2014;12(1):26. https://doi.org/10.1186/1478-4491-12-26

5. Castro-Leal F, Dayton J, Demery L, Mehra K. Public spending on healthcare in Africa: Do the poor benefit? Bull World Health Organ 2000;78(1):66-74. https://doi.org/10.1093/wbro/14.1.49

6. Ataguba JE-O, Akazili J. Health care financing in South Africa: Moving towards universal coverage. Cont Med Educ 2010;28(2):74.

7. Tierney WM, Miller ME, McDonald CJ. The effect on test ordering of informing physicians of the charges for outpatient diagnostic tests. N Engl J Med 1990;322(21):1499-1504. https://doi.org/10.1056/
chas charges for outpatient diagnostic tests. N Engl J Med 1990;322(21):1499-1504. https://doi.org/10.1056/ NEJM199005243222105

8. Tek Sehgal R, Gorman P. Internal medicine physicians' knowledge of healthcare charges. J Grad Med Educ 2011;3(2):182-187. https://doi.org/10.4300/JGME-D-10-00186.1

9. Bade K, Hoogerbrug J. Awareness of surgical costs: A multicenter cross-sectional survey. J Surg Educ 2015;2(1):23-27. https://doi.org/10.1016/j.jsurg.2014.06.017
10. Allan GM, Innes G. Family practice residents' awareness of medical care costs in British Columbia. Fam Med 2002;34(2):104-109.

11. Glickman L, Bruce EA, Caro FG, Avorn J. Physicians' knowledge of drug costs for the elderly. J Am Geriatr Soc 1994;42(9):992-996. https://doi.org/10.1111/j.1532-5415.1994.tb06594.x
Gericts for

12. Schilling UM. Cost awareness among Swedish physicians working at the emergency department. Eur I Echilling UM. Cost awareness among Swedish physicians working at the emergen

13. Bailey CR, Ruggier R, Cashman JN. Anaesthesia: Cheap at twice the price? Anaesthesia 1993;48(10):906909. https://doi.org/10.1111/j.1365-2044.1993.tb07428.x

14. Long MJ, Cummings KM, Frisof KB. The role of perceived price in physicians' demand for diagnostic tests. Med Care 1983;21(2):243-250.

15. Covington MF, Agan DL, Liu Y, Johnson JO, Shaw DJ. Teaching cost-conscious medicine: Impact of a simple educational intervention on appropriate abdominal imaging at a community-based teaching hospital. J Grad Med Educ 2013;5(2):284-288. https://doi.org/10.4300/JGME-D-12-00117.1

16. Sommers BD, Desai N, Fiskio J, et al. An educational intervention to improve cost-effective care among medicine housestaff: A randomized controlled trial. Acad Med 2012;87(6):719-728. https://doi. org/10.1097/ACM.0b013e31825373b3

17. Fairbrass MJ, Chaffe AG. Staff awareness of cost of anaesthetic drugs, fluids, and disposables. BMJ 1988;296(6628):1040. https://doi.org/10.1136/bmj.296.6628.1040

18. Hernu R, Cour M, de la Salle S, Robert D, Argaud L. Cost awareness of physicians in intensive care units: A multicentric national study. Intens Care Med 2015;41(8):1402-1410. https:// /doi.org/10.1007/ s00134-015-3859-1

19. Ryan M, Yule B, Bond C, Taylor RJ. Scottish general practitioners' attitudes and knowledge in respect of prescribing costs. BMJ 1990;300(6735):1316-1318. https://doi.org/10.1136/bmj.300.6735.1316

Accepted 1 June 2017. 